\title{
Chapter 11 \\ Enlightened Leadership: Coping with Chaos in Increasingly Turbulent Times
}

We want to summarize and thereby tie together all of the previous discussions on Mental Health, Attachment Theory, Inquiring Systems, Culture, the Jungian Framework, Conflict Management, Surfacing Assumptions, and Defense Mechanisms. Used appropriately, they are indispensable in helping people deal with Reality.

Above all, coping with chaos requires Enlightened Leadership. If we're to improve our institutions and society as a whole, nothing less will suffice.

\subsection{The Problem Management Organization (PMO)}

Since the early 1970s, Kilmann has worked with scores of companies, both domestic and international, to help them address their most complex problems. To accomplish this, the organizations have had to bring together diverse groups of experts and representatives with very different points of view to address the "Big Picture." As a result, it's been necessary to create a new institutional arrangement, the Problem Management Organization or PMO for short. ${ }^{1}$

In particular, if we are to have any chance whatsoever of coping with Wicked Messes, then PMOs are an absolute necessity. Most basic of all, a PMO cannot accomplish its goals without Enlightened Leadership both to initiate and to see the entire process through. Without it, Wicked Messes cannot be successfully addressed. As a result, the contrast between Enlightened Leadership and Malignant Leadership couldn't be greater.

If the leaders in society are suffering from Mental Illness and other forms of malignancy, they will persist in advocating — and worse yet, implementing — simplistic, political, self-serving, or harmful solutions to complex problems.

\footnotetext{
${ }^{1}$ Kilmann, R. H. Quantum Organizations: A New Paradigm for Achieving Organizational Success and Personal Meaning (Newport Coast, CA: Kilmann Diagnostics, 2011).
} 
In brief, The worst Mess of all is Malignant Leadership. It's worse because Leadership is a critical component, if not the most important ingredient, of every Wicked Mess. Dysfunctional behavior of any kind prevents an organization, institution, or nation from effectively addressing Mega Crises. Indeed, Malignant Leadership adds even more chaos to what is already a highly chaotic situation. Thus, for better or worse, Leadership is deeply intertwined with every System. As a result, the enormous challenge before us is to do all that we can to ensure that Enlightened Leadership is in place before any organization, institution, or nation attempts to address Wicked Messes, especially those whose life-and-death consequences require immediate attention.

To begin with, it's vital to identify the kinds of expertise and perspectives that are necessary to bring to bear on the unbelievably complex, messy problems with which we are confronted. In the case of the unprecedented Wicked Messes we are facingthe Pandemic, Political Divisiveness, Waves of Protests, Police Violence, Systemic Racism, Global Warming, and a Floundering Economy-making effective use of experts with very different backgrounds and perspectives is absolutely essential if we are to make any headway at all on these and all of the other momentous problems we face. PMOs require the best experts in Public Health, Infectious Disease, Alternative Health, Economics, and so on. We also need to include those who are deeply affected such as Public School Principals and Teachers, College Administrators and Professors, Students, Parents, Psychologists, Lawyers, Politicians, Police Officers, Business Leaders, and a diverse group of Community Leaders from urban and rural settings. In addition, it requires people who differ with regard to gender, race, ethnicity, religion, and sexual orientation.

While it would seem that the vital need for such a wide range of expertise (and different demographics) requires a large group, in practice 30 to 40 diverse participants are usually more than sufficient for a PMO.

Ideally, all the participants would be Secure Adults and therefore have largely dealt with whatever traumas they've suffered as children. In the best case, they will have developed more resourceful, Reality-based beliefs, attitudes, and behaviors. The will also have (a) a healthy ego; (b) the ability to communicate their ideas to others in a civil, dignified, and engaging manner; and, most importantly, (c) the interpersonal skills to listen intently - and earnestly-when others are presenting different facts, theories, and opinions.

Once a group of about 30 to 40 diverse experts and representatives have been assembled, the next step is to develop a beneficial "behavioral infrastructure." It's absolutely essential to ensure a PMO's healthy behavior. It consists of three Tracks: the Culture Track, the Skills Track, and the Team Track. ${ }^{2}$

In most cases, it takes about three full days of instruction and group exercises in order to instill an effective behavioral infrastructure. Nonetheless, the 3-day investment in time and education is the only way to ensure that the members will be able to have a productive and probing dialogue across very different perspectives.

\footnotetext{
${ }^{2}$ op. cit., Kilmann, R.H.
} 


\subsection{The Culture Track}

As we discussed in an earlier chapter on the need to change the culture of Policing, we cannot overemphasize the importance of surfacing the actual behavioral normsthe unwritten "rules of the game" that govern "how we do things around here"-so it can be determined if they are functional, and thereby healthy or not.

Consider the cultural norm that says, "Respect only those persons and perspectives that support your particular background, experience, and training, official company policies, and consider all other viewpoints as irrelevant to the problem at hand." If such a norm is operating, it prevents members from reaching more encompassing — and thereby synergistic — approaches to Wicked Messes.

Once the actual, and typically dysfunctional, cultural norms have been surfaced, the focus shifts to specifying the desired, more functional norms that would make full use of a PMO's collective expertise and wisdom. For example: "Since all of us are limited in our knowledge and experience to be expert in every aspect of a Wicked Mess, we not only need to respect vastly divergent viewpoints, but we explicitly need to include them in our discussions."

The differences between the actual and desired norms identify the "culture-gaps" that must be significantly overcome before an effective behavioral infrastructure can be established.

But how can the culture-gaps be closed? Once they are out in the open for explicit examination, the participants need to determine from this point forward, how they will reward the new desired behaviors. They need to specify the positive sanctions that will be administered whenever the members enact the new desired norms as well as what negative sanctions will be if there is any reappearance of the old, dysfunctional norms.

Simply put, if the members do not experience any positive consequences when they enact an agreed-upon desired norm and if they also do not experience any negative consequences when they act out what their group has identified as a dysfunctional norm, why would they ever be motivated to change?

Essentially, once they have openly agreed to do it, it's the basic social power that a group has over its individual members that enables it to maintain a healthy culture. Thus, instead of allowing the previous silent sanctioning system to reinforce old dysfunctional behaviors, a PMO intentionally designs a Legal, Ethical, and Open Sanctioning System that ensures a healthy exchange of ideas and knowledge.

\subsection{The Skills Track}

Once the members of a PMO have identified the culture-gaps and have designed an effective sanctioning system for closing them, it's necessary to proceed with the Skills Track. The central task for the entire community is to learn the five steps of effective problem management: (1) sensing important problems (i.e., recognizing 
and acknowledging problems in the first place), (2) defining them correctly (i.e., making sure that one is solving the "right problems" vs. the "wrong ones"), (3) deriving workable solutions, (4) implementing them effectively, and then (5) evaluating the outcomes. ${ }^{3}$

When the members have learned the key lessons that are embedded in the five steps of problem management, they are then able to understand why any truly complex problem cannot possibly be defined and solved by relying on only one area of expertise, scientific discipline, or perspective. Since humans have limited mental capacities that more often than not lead them to become extreme specialists, each of us can only deal with a few facts and theories at a time. As a result, it's virtually impossible for any one person, no matter how brilliant he or she is, to be equally expert in Public Health, Medicine, Police Work, Politics, Pandemics, Psychology, Climate Change, Economics, Law, Biology, and Leadership, let alone to fully appreciate the different life experiences — and hence, the needs and concerns - of people with different racial, ethnic, religious, generational, and other backgrounds.

Yet, until we can successfully integrate the divergent perspectives of different experts and life experiences, we will always fail to accurately define the root causes of complex problems. Anyone, let alone a leader, who proclaims, "I alone can fix it," is only fooling himself or herself and all those who allow themselves to be conned into believing that a single perspective is sufficient for effectively addressing Wicked Messes.

A second critical component of the Skills Track is Assumptional Analysis. If a person's assumptions are blatantly false, then his or her conclusions-i.e., how he or she defines the root causes of a problem - are also blatantly false. Ironically, since one has to take a great deal of things for granted in order to become an expert, most experts are blind to their own assumptions. But what one person takes as a given, another regards as an unwarranted assumption. Every discipline or specialization has a set of behind-the-scenes assumptions that are automatically and unconsciously treated as facts. Indeed, assumptions govern the ways in which an expert is taught to see the world. As a result, no specialization can exist without automatically accepting a set of assumptions that are specific to a particular discipline.

For this reason, the Skills Track not only teaches, but reinforces the participants' use of a step-by-step method for surfacing one's typically hidden and underlying assumptions, analyzing whether they are in fact true or false, and then based on further group discussions, probing investigations, and survey research, revising any false assumptions. As various assumptions are modified, the group's initial conclusions will be as well. Clearly, it's essential that any and all approaches to managing Wicked Messes be based on valid assumptions, not on past falsehoods.

\footnotetext{
${ }^{3}$ Kilmann, R. H. "Problem Management: A Behavioral Science Approach.” In G. Zaltman. Ed., Management Principles for Nonprofit Agencies and Organizations (New York: American Management Association, 1979), 213-255.
} 


\subsection{The Team Track}

The purpose of the Team Track is to learn how to have productive group meetings by applying several, agreed-upon key principles. At the beginning of each meeting, everyone needs to be fully aware of the objectives. Members need to plan their time wisely and determine the priority of agenda items before any subject is discussed at length. Members must address the most important issues first and the less important ones last. Participants should also plan how each agenda item will be approached and whether it can be subdivided into several manageable pieces so a problem's inherent complexity does not immobilize them. (Needless to say, with regard to Wicked Messes, this poses special challenges.) Spending time first planning how to apply key principles saves a lot of time later.

Furthermore, the more talkative members of the group need to make a concerted effort to bring the less talkative ones into every discussion to ensure that all perspectives are heard and that all of the available information is used. Even more to the point, the members need to assess regularly whether the group's cultural norms continue to support new and creative ideas. All communications need to be civil, thereby showing respect for every person. Only one person should speak at a time. Everyone else needs to listen. Collaborative behavior, not competitive behavior (fighting to talk the most and trying to win the final argument), needs to be the desired norm in order to make full use of everyone's knowledge and thereby help to ensure the best outcomes. Occasionally, the members need to take a break from the discussion on content and concentrate on the process: "How are we doing as a group? Are we applying all the key principles and practices for having a productive meeting? If not, what should we be doing differently, and better?"

When it comes to ensuring that the members will actually apply the key principles and thus continuously improve their group's process, it's beneficial to appoint a "process observer" (PO) at the start of every meeting. The PO is responsible for monitoring how well the key principles are actually guiding the group's discussions. At the end of each meeting, the PO summarizes what the group did well and in the ways it fell short. A plan is then formulated regarding what can be done to improve the next meetings.

As we've said, in most cases, a diverse group of 30 to 40 participants can learn the material in the Culture, Skills, and Team Tracks in about 3 days. Nonetheless, someone always asks: "Wouldn't it be better to bypass those three days of 'schoolwork' and, instead, use that same amount of time to begin addressing a particular Wicked Mess?"

Basically, without a healthy behavioral infrastructure at the beginning, the members would be wasting their time and, most importantly, would fail in coping with a Wicked Mess that, by definition, exceeds every person's limited perspective, knowledge, and experience. 


\subsection{The Jungian Framework}

Once an effective behavioral infrastructure has been established, based on the four Jungian Types, the 30 to 40 participants are divided into four groups: ST, NT, SF, and NF. The four Types guarantee that any Wicked Mess will be approached in four radically different ways. Based on past experience, the various academic and professional specialties and disciplines will be spread across the four Jungian groups. Nonetheless, there is a strong tendency for Economists to be mostly ST and NT and for Psychologists, especially Clinical, to be mostly SF and NF, etc.

As we've noted, the Jungian Framework is based on two different ways of gathering information, indeed what is considered to be "information," (Sensing and Intuiting) and two different ways of making decisions (Thinking and Feeling). Thus, each group slants its perspective toward the extreme characteristics of its Personality Type, which vary along the lines of Technical vs. Human and Short-Term vs. Long-Term.

Briefly, the ST group, no matter what the particular specialties it contains, will focus on the Short-Term Technical aspects of a complex problem; the NT group will examine on the Long-Term Technical aspects of Wicked Messes; the SF group will give most attention to the special concerns of particular families and neighborhoods; and the NF group will mainly consider the Long-Term consequences to society and the human condition as a whole.

Once the participants have been placed into one of the four Jungian groups, typically based on the Myers-Briggs Type Indicator ${ }^{4}$ or a similar instrument for assessing the same four Psychological Styles, ${ }^{5}$ each group is instructed to meet, introduce everyone to one another, and then openly discuss and confirm its desired cultural norms. It next confirms its knowledge of problem management and Assumptional Analysis. It then affirms that it will make use of a PMO for ensuring that all the key principles of group process will govern how members interact with one another during every meeting.

Next, each of the four Jungian groups plans the process by which it will develop its initial conclusions: its position statement on the "the primary root causes of a Wicked Mess" and what to do about it. Each group then uses Assumptional Analysis to surface its underlying assumptions, which are then sorted into a matrix according to the certainty/uncertainty of an assumption being true or false as well as the relative importance of each assumption support for the group's initial conclusions about the root causes of a Wicked Mess, in a word, defining the problem. Naturally, exactly how the problem is defined subsequently constrains the options that will be considered for how a particular complex problem can be best addressed.

Once each of the four Jungian groups has developed its initial conclusions with regard to the root causes of a Wicked Mess, the stage is set for an intense debate.

\footnotetext{
${ }^{4}$ Myers, I. B. Myers-Briggs Type Indicator (Mountain View, CA; CPP, Inc., 1962).

${ }^{5}$ Kilmann, R. H. Kilmanns Personality Style Instrument (Newport Coast, CA: Kilmann Diagnostics, 2011).
} 
Each group in turn presents its initial conclusions and its underlying assumptions to the entire community. The other three groups are allowed to question - and debatethe truth and certainty of the focal group's underlying assumptions behind its initial conclusions.

After each group has presented its case to the entire community, followed by discussions and debates with the other three groups, a list is made of all the "unresolved issues."

Typically, during the four intergroup debates, a number of modifications to the conclusions and assumptions will already have been made. But even if the debates are completely open, candid, and thorough, there will still be some basic core issues that remain unresolved. Indeed, lingering conflicts get at the heart of why Messes are so complicated and overwhelming. As a consequence, another mechanism is needed to help resolve the unresolved conflicts that remain among the four groups in order that the entire community can use all of its collective knowledge and wisdom to develop the most comprehensive, inclusive, and promising approach to the problem, i.e., Wicked Mess, at hand.

\subsection{S-Groups}

A new and very different kind of group is formed from the entire PMO. It's called a Synthesis or S-Group. The S-Group is made up of two members from each of the four previous Jungian groups. Ideally, each of the selected S-Group members has a balanced repertoire of conflict-handling behavior, as measured by the ThomasKilmann Conflict Mode Instrument. ${ }^{6}$ In this way, an S-Group is able to effectively use all five approaches to conflict management: competing, collaborating, compromising, avoiding, and accommodating.

In essence, the unresolved issues represent the "nagging underlying conflicts" that cut across the radically different disciplines and perspectives that could not be resolved through the intense discussions and debates among the four Jungian groups. The goal now is to address and resolve the remaining differences with the use of one or more conflict-handling modes.

In the best S-Groups, collaborating prevails. This enables the members to incorporate fully the different perspectives into an integrated approach. When this is not possible, compromising may be able to allow each expert's perspective and assumptions to be partially incorporated into an agreed-upon approach to a particular Wicked Mess. In some cases, one expert is clearly more knowledgeable than the others so he or she needs to assert their position, and hence use competing to get their points across. Naturally, some members in an S-Group will choose to accommodate other people's perspectives, especially when the latter clearly know more

${ }^{6}$ Thomas, K.W., and R. H. Kilmann. The Thomas-Kilmann Conflict Mode Instrument (Sunnyvale, CA: Xicom and The Myers-Briggs Company, 1974). 
about a particular topic or area of expertise. Other members will sometimes choose to avoid debating certain topics that turn out to be much less important than first thought. The effective use of the five different approaches for managing conflict is precisely why it's so important for each member in the S-Group to have a balanced repertoire of conflict-handling behaviors.

It's often beneficial to form a number of S-Groups, from two to four, each composed of two representatives from the previous Jungian groups. This allows for greater participation by all of the members of a PMO, instead of relying on only a handful to form a single S-Group.

In most cases, when multiple S-Groups are used, each of the resulting syntheses is not that different from one another, especially since each $S$-Group has been purposely composed by choosing two participants from each of the same four Jungian groups. In fact, it's a powerful experience for the members to see that the multiple $\mathrm{S}$-Groups are in basic agreement regarding (a) how to resolve the unresolved issues/ conflicts and (b) how to derive a new, holistic conclusion that takes into account all of the relevant disciplines and perspectives.

Not surprisingly, a striking similarity across the revised conclusions that were derived by multiple S-Groups serendipitously confirms the validity of the whole process. Unless there are logistical constraints, we strongly encourage the use of multiple S-Groups to address the unresolved conflicts of the four Jungian groups.

Another serendipitous byproduct that usually arises from an enlightened problem management process is as follows: Once a large gathering of diverse experts and relevant representatives have intimately experienced an effective PMO in action, some of the members naturally wonder (often out loud): "What would our society be like if all families, neighborhoods, schools, work organizations, and federal, state, and local governments had already learned how to create mentally healthy and Reality-based behavioral infrastructures so that the following PMO skills would be widely shared-and practiced — throughout our social systems: (1) identifying and closing culture-gaps, (2) using the five steps of problem management along with Assumptional Analysis, and (3) applying the key principles of group process during all discussions and meetings?"

The short answer is as follows: Widespread use of effective PMOs throughout society (from families to the United Nations) would be a major step in helping to transform our current divisiveness (with its corresponding animosity across most demographic and national boundaries) into illuminating debates and fully integrated solutions for effectively resolving Wicked Messes. ${ }^{7}$ Such a widespread transformation of all of our social systems is not just a lofty ideal toward which to strive, but it also becomes a Moral Imperative for the healthy evolution of the human race.

\footnotetext{
${ }^{7}$ Kilmann, R. H. The Courageous Mosaic: Awakening Society, Systems, and Souls (Newport Coast, CA: Kilmann Diagnostics, 2013), Chapter 20.
} 


\subsection{The Psychodynamics of Leadership in a PMO}

We return to the fundamental theme of the book: What kind of Leadership is required to initiate, conduct, and support the entire PMO process-effectively, legally, and ethically - in the best interests of society and the entire planet?

In the worst cases, if the leaders are Insecure with Mental Health challenges of one kind or another, they won't tolerate, let alone respect, different points of view. Nor will they be inclined to recruit a diverse group of participants. Typically, Insecure and mentally challenged leaders will do their best to prevent alternative perspectives from coming to light, particularly any opinions that even remotely appear to contradict the leader's personal and political agenda. We cannot say it enough: There is no more perfect example of Malignant Leadership than when a top leader proclaims again and again: "I alone can fix it."

The leaders also need to appreciate the importance of creating and maintaining a healthy behavioral infrastructure so that all of the diverse talent in a PMO will rise to the surface for open discussion and debate, and not remain submerged and inaccessible. Having brilliant and experienced experts in the same room is a complete waste of time if the operating cultural norms and implicit sanctioning system punish people who even dare to express views that are different from a leader's opinions and/or egocentric patterns of thought. Regardless of a member's brilliance in a particular discipline or perspective, there's no substitute for also having to learn the fundamentals of problem management and Assumptional Analysis, both of which are rarely taught in professional schools. Moreover, experts are rarely required, or take the time on their own, to learn the skills necessary for engaging in civil, dignified, and open-minded conversations. One of the prime purposes of a PMO is to correct such deficiencies in our formal educational system.

The top leaders also need to understand that a special effort is needed to create effective teamwork among the diverse members of a community so that cooperation, not competition, will rule the day. Without following the key principles of effective group process, all the talent in the world cannot-and will not-be utilized. For example, if a few members dominate the group discussion and thereby silence the quieter members, some of the valuable wisdom of the group as a whole will - inadvertently or deliberately — be excluded from the discussion. Every member in a Jungian group or an S-Group needs to have his or her voice heard.

We return to the core question: What exactly is Enlightened Leadership? Ironically, the prime leader does not have to possess any particular scientific, professional, or in-depth knowledge about the complex issues and Wicked Messes in question. Of course, what the leaders do know about the various relevant subjects and scientific disciplines will enable them to better appreciate what the experts have to say, and why. But the leader's knowledge, or lack thereof, about the various topics should not be the basis for judging his or her success as a leader.

Instead, the essential quality by which to judge the success of the top leader of a nation or institution with regard to addressing any and all Wicked Messes can be succinctly summarized as follows: 
The top leader and his or her leadership team needs to make absolutely sure that a diverse group of experts and other representatives will engage in a Realitybased, mentally healthy process that has the best chance of effectively addressing the entire scope of a Wicked Mess, both short-term and long-term.

To be absolutely clear, we are far less concerned about the depth and breadth of a leader's scientific knowledge or prior work experiences with regard to all of the aspects of a Wicked Mess. Instead, we are most concerned about a leader's ability to initiate a healthy and resourceful process that will greatly increase the likelihood that a Wicked Mess can be managed effectively. So long as the leader initiates the process and also models the very behavior he or she wishes to see occur within a $\mathrm{PMO}$, an Enlightened Leader is doing exactly what's needed for everyone to survive and thrive in today's global village.

In sharp contrast, the exact opposite of Enlightened Leadership is Malignant Leadership. It ensures that all Wicked Messes will increasingly undermine, and ultimately destroy, the soul of democracy and the hope for health and happiness.

Malignant Leadership only fuels more and more chaos, which makes everything far worse. Malignant Leadership, and all those who support it, will continue to escalate and prolong every Wicked Mess.

Alternatively, Enlightened Leadership mobilizes-and inspires-a mentally healthy, Reality-based PMO, which thus provides a thoroughly diverse community of participants with the best chance of effectively addressing the onslaught of mega challenges to democracy and civilization.

Given the increasing prevalence of Wicked Messes, more than ever before, we need Enlightened Leaders.

Open Access This chapter is licensed under the terms of the Creative Commons Attribution 4.0 International License (http://creativecommons.org/licenses/by/4.0/), which permits use, sharing, adaptation, distribution and reproduction in any medium or format, as long as you give appropriate credit to the original author(s) and the source, provide a link to the Creative Commons license and indicate if changes were made.

The images or other third party material in this chapter are included in the chapter's Creative Commons license, unless indicated otherwise in a credit line to the material. If material is not included in the chapter's Creative Commons license and your intended use is not permitted by statutory regulation or exceeds the permitted use, you will need to obtain permission directly from the copyright holder.

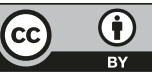

